\title{
LATERAL SPREAD OF LIGHT ADAPTATION IN THE RAT RETINA ${ }^{1}$
}

\author{
Daniel G. Green, lillian Tong and Carol M. Cicerone \\ Vision Research Laboratory, University of Michigan, Ann Arbor, MI 48109, U.S.A.
}

(Received 1 June 1976; in revised form 22 August 1976)

\begin{abstract}
Recordings from the rat optic tract fibers were used to assess changes in sensitivity under various conditions of adaptation. An adapting background which excites only a small fraction of the rods can yet cause a several-fold change in sensitivity. A small adapting spot much more effectively decreases the cell's sensitivity to a superimposed test than to test spots in positions far from the adapting locus. Thus, adaptation spreads laterally but not uniformly throughout the ganglion cell center. Scattered light does not account for the spread, since a displaced adapting spot can be more effective than one superimposed on the test spot.
\end{abstract}

\section{INTRODUCTION}

Tremendous advances in our understanding of the well-known changes in sensitivity occurring during light adaptation have been made in recent years. The old hypothesis of Hecht (1937) that an adapting background changes sensitivity simply because it bleaches away the photosensitive pigment has been shown to be incorrect, since much larger changes in sensitivity occur than can be explained by the small amounts of rod pigment that have been bleached (Rushton, 1961; Dowling, 1963). In fact, at scotopic levels, the desensitizing effect of a background light is almost completely independent of the amount of rhodopsin bleached (Rushton, 1965a; Dowling, 1967). This has been shown in two different ways. First backgrounds elevate increment thresholds according to the WeberFechner law at low levels where no measurable bleaching occurs. Second, thresholds remain constant under conditions where larger and larger amounts of rhodopsin are being bleached. Within seconds after the onset of an adapting background, increment thresholds in the rat eye reach equilibrium (Green, 1973). Yet, rhodopsin regenerates so slowly in the rat eye that a dim background will continue to bleach small, but successively larger, amounts of pigment for periods of $300 \mathrm{sec}$ and longer after the onset of a steady adapting stimulus (Dowling, 1963).

The rather convincing demonstration from several laboratories that adaptive effects can spread laterally across the retina (Lipetz, 1961; Rushton and Westheimer, 1962; Rushton, 1965b; Easter, 1968; Cleland and Enroth-Cugell, 1968) seemed to rule out the possibility that the sensitivity changes occurring in adaptation originated in the photoreceptors. Illumination in one area of the retina was shown to elevate thresholds for test stimuli falling on areas which had not been directly exposed to light. These experiments seemed to establish that changes in retinal sensitivity are controlled by signals pooled from many receptors.

However, Boynton and Whitten (1970) used the

${ }^{1}$ A preliminary report of these results (Green and Tong, 1975) was presented to the annual meeting of ARVO, April, 1975. receptor potential of the monkey fovea to show that an adapting background causes primate cones to change their sensitivity. Other experiments, using sodium asparate to isolate receptor potentials, have provided ample evidence that skate rods (Dowling and Ripps, 1972), goldfish rods and cones (Witkovsky, Nelson, and Ripps, 1973), and frog rods and cones (Hood and Hock, 1973; Hood, Hock and Grover, 1973) display the full range of adaptive phenomena. The results of intracellular recordings from single photoreccptors also demonstrate that receptors can contribute significantly to the sensitivity changes measured in more proximal neurons (Grabowski, Pinto and Pak, 1972; Kleinschmidt, 1973; Baylor and Hodgkin, 1974; Kleinschmidt and Dowling, 1975). Finally, the experiments of Rushton and Westheimer (1962) and Rushton (1965b), demonstrating psychophysically that adaptation spreads laterally through the summation pool, have been repeated and extended by Barlow and Andrews (1967, 1973), who argue for narrower spread of adaptive effects.

The experiments we report here were undertaken as an attempt to reconcile these new results with the older evidence for pooling of adaptive signals. One critical problem is that optical aberrations, scatter in the ocular media and back scatter from the retina, will invariably cause light to spread outside the geometric image of the target on the retina. Consequently, even if the appartus controlling retinal sensitivity were entirely in the photoreceptors, light from an adapting stimulus would be expected to elevate the threshold of a laterally placed test stimulus. Demonstrating that adaptation spreads laterally is, in itself, insufficient to establish the site of visual adaptation; one must separate neural spread from the possible effects of stray light.

Working on the cat, Bonds, Enroth-Cugell and Pinto (1972) have approached this problem by measuring the double pass optical spread function and have used this to estimate the quality of the retinal image. They find that the optical spread function is narrow in comparison with the dimensions of the ganglion cell receptive fields. Parallel studies provide strong evidence for non-rcceptor adaptation by showing areal summation of adaptive effects over the com- 
plete ganglión cell recoptive field center /Cleland and Enroth-Cugell, 1968; Enroth-Cugell and Shapley, 1973). We report here that, while in general we confirm their conclusions about neural pooling of adaptive signals, we find in the rat that there is not complete pooling of adaptation over the central mechanism of the ganglion cell receptive field.

\section{METHODS}

Rats (Long Evans) were raised in dim illumination and dark-adapted for $12 \mathrm{hr}$ or more. They were initially anesthetized with urethane $(200 \mathrm{mg} / 100 \mathrm{~g})$ intraperitoneally with subsequent small doses as needed. All surgery was performed under dim, red illumination. The rat was mounted in a Baltimore Instruments stereotaxic apparatus. A hole was drilled in the skull to the right of midline and included $0.5 \mathrm{~cm}$ on either side of the bregma. The dura was reflected. The upper cyclid of the left eye was removed and the conjunctiva was severed from the globe. Care was taken to keep the comea moist. In early experiments, the left eye was then stablized with a semi-eye ring similar to that used by Enroth-Cugell and Shapley (1973). A halfring, designed to fit snugly around the eveball, was attached to a long rod that could be held rigidly to the stereotaxic frame. Eastman 910 Adhesive was applied to the ring which was then lowered onto the upper half of the eyeball. Care was taken to keep the glue off the cornea. More recently, we have sewn a full-ring to the conjunctiva using silk sutures.

The pupil was dilated with atropine sulphate $1 \%$. We found it very difficult to accurately refract our animals and, in fact, usually found them to be about $10 \mathrm{D}$ hyperopic when refracted by streak retinoscope. There are several good reasons for believing that this error is more apparent than real. Glickstein and Millodot (1970) argue that reflected light comes from the inner surface of the retina so that in a small eye, the finite thickness of the retina causes a large apparent hyperopic refractive error. Confirmation of this point of view comes from Wiesenfeld and Branchek's (1976) recent behavioral studies showing rats are actually myopic. Consequently, we attempted to select a contact lens which left the original uncorrected hyperopic refractive error unchanged. Finally, a black contact lens, plano, with a clear pupil. $0.5-1.0 \mathrm{~mm}$ dia, was placed on the eye. By using a small pupil one can increase the depth of the focus and thereby minimize the effects of any remaining uncorrected refractive errors. Calculation shows that with a $1 \mathrm{~mm}$ artificial pupil, the rat eye can tolerate about $10 \mathrm{D}$ of refractive error before the size of the blur circle would start to approach that of our smallest ganglion cell receptive fields.

On a typical animal, an electrode positioned $1.5-2.5 \mathrm{~mm}$ lateral and at bregma AP would pass through the optic tract if lowered about $8 \mathrm{~mm}$ from the surface of the brain. The optic tract was first located by using a glass pipette microelectrode with a tip of about $5-10 \mu \mathrm{m}$ filled with low melting point metal (Cerrolow 136), plated with platinum. A ping-pong ball was placed over the left eye of the rat and illuminated uniformly in brief, dim flashes. Units in rat optic tract fired in response to this diffuse stimulus. Once the optic tract was located, a smaller tipped cerrolow electrode or tungsten-wire-in-glass electrode (Levick, 1972) was lowered at the same coordinates in order to better isolate single units. After a single unit had been isolated, the ping-pong ball was removed and the receptive field was carefully localized on a tangent screen. The position of the stimulus was marked on the reverse side of the screen. A two-channel stimulator allowed two spots (test and adaptation) to be independently varied in size, position, and intensity. The test and adapting stimuli were derived from a $100 \mathrm{~W}$ solid filament tungsten lamp (GE) and a $150 \mathrm{~W}$ xenon arc lamp (Osram), respectively. Using the unit's response as the criterion. the test spot was centered approximately in the receptive field. Fine adjustment were made by successively moving the test spot until a position was found such that it small laterally-placed adapting spot had equal effect when placed in each of four symmetrically located positions around the test spot. This seemed to be an cxecedingly sensitive procedure for locating the receptive field center.

To quantify the effect of various experimenta! manipulations on the ganglion cell responses, we used threshold measures of sensitivity. That is. the intensity of the test flash was adjusted until the unit gave a criterion response. The response to a test flash was evaluated by listening to the spike discharge on a loudspeaker and by viewing a response dot pattern on a storage oscilloscope (Tektronix, type 564). The oscilloscope trace starts across with the onset of each test flash. Each spike is detected by the trigger circuitry of an oscilloscope (Tektronix, type 565) and converted to a dot by applying the pulses to the $Z$-axis of the storage oscilloscope. In addition, a low-frequency triangular wave form is applied to the oscilloscope's vertical amplifier, causing the responses to each successive presentation of the stimulus to be displaced downward on the screcn. The accumulation of dots represents the unit's response to multiple flashes

The dot patterns in Fig. I show a typical intensityresponse series from an "off" unit. The stimulus was a small (approx $1^{1}$ ) test spot centered in the unit's receptive field. The test flash was made successively brighter in 0.3 $\log$ unit steps. Each doubling of stimulus intensity causes the response discharge to become stronger. In determining a threshold response, the stimulus intensity was adjusted until the dot pattern looked approximately like the $\times 2$ pattern in the upper right-hand corner. Usually, repeated determinations of threshold were within 0.1 or, at most, $0.2 \mathrm{log}$ units of each other. The results to be reported here are from "of" units even though "on" and "of" units are found with about equal frequency. Most "on" units responded tonically to adapting spots placed within the receptive field area. With such units, it was nearly impossible to detect incremental responses using our auditory and visual display methods.

\section{RESLLTS}

\section{Pooling of adaptation and excitation}

One approach to showing that changes in retinal sensitivity are produced by signals pooled from many receptors is to demonstrate that the adaptive signals are pooled spatially with the same sensitivity profile as the ordinary response receptive field. For example, Enroth-Cugell and Shapley (1973) conclude that the central response mechanism of cat retinal ganglion cells act as a unit, adding signals from many photoreceptors to produce responses and adding adaptive signals to set the sensitivity of the center.

Figure 2 shows a set of ganglion cell responses which demonstrate that excitation and adaptation are not pooled in this way in the rat retina. The experiment went as follows. Two equally sensitive positions on the plateau of the receptive field were located (Figs. 2a and b). The test stimulus was increased tenfold (Fig. 2c) and a small, steady adapting light was placed on top of it. This adapting stimulus was adjusted to bring the test light to about threshold (Fig. 2d). When the test spot was moved to the other position, it became clear that the adapting spot has a much weaker effect on a laterally-placed test spot (Fig. 2e). In this displaced position, the test intensity had to be reduced by a factor of 2.5 to produce a threshold response (Fig. 2f). 


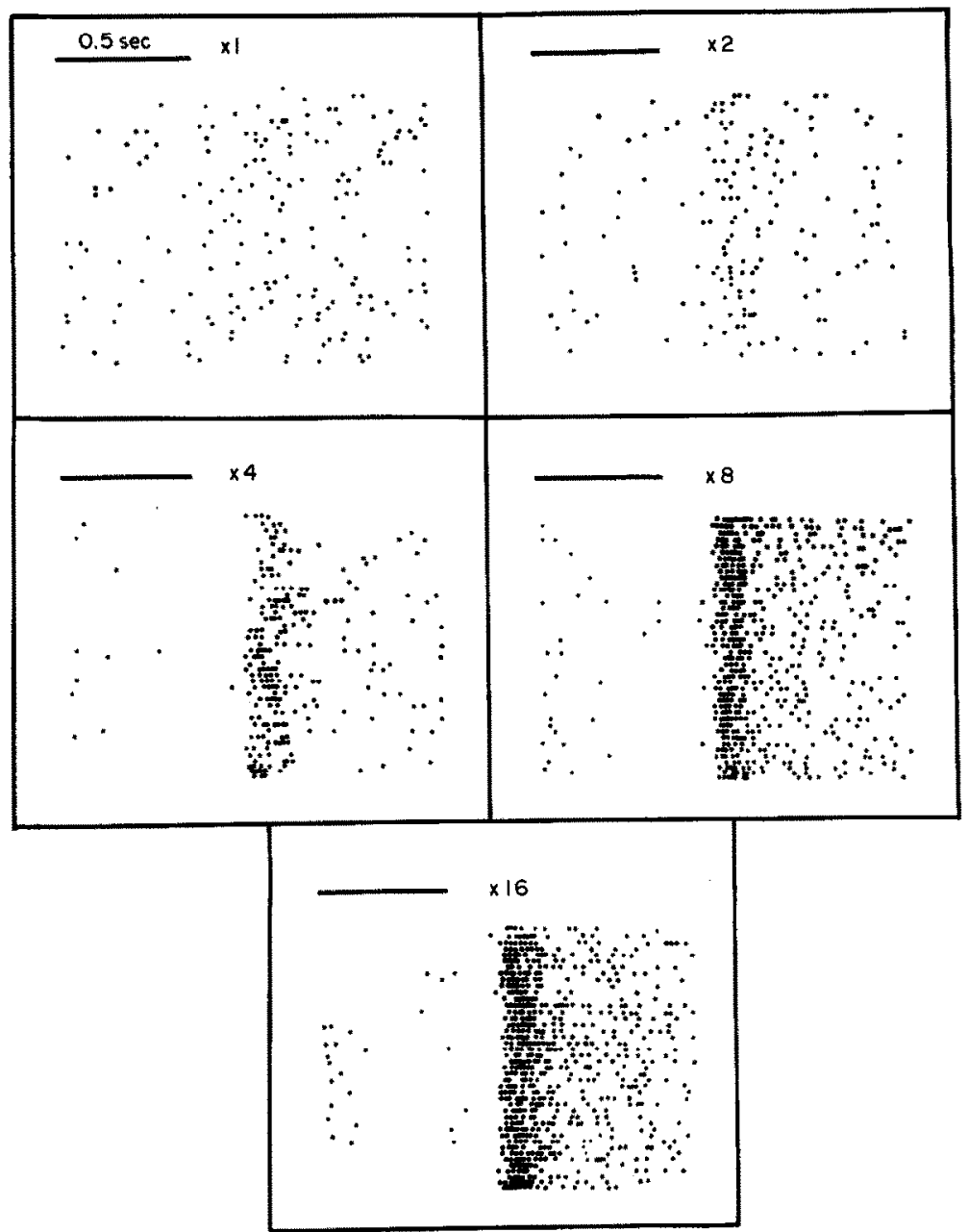

Fig. 1. Dot-displays illustrating the responses of a typical "of" unit to a series of stimuli increased in $0.3 \mathrm{log}$ unit increments. The dot-display labelled $\times 2$ illustrates a level of response which is close to "threshold". In this and subsequent experiments, the duration of the test flash was $0.5 \mathrm{sec}$. The time interval between successive flashes was $2.0 \mathrm{sec}$.

These findings have been quantified by comparing the excitation receptive field (ERF), the sensitivity profile measured by moving a test flash across the field, with the sensitivity profile measured in the presence of a small, steady adapting spot, $A$ (ERF/A), for the same unit. This experiment is shown in Fig. 3. The excitation receptive field of the "off" unit shown in Fig. 2 has been plotted as a function of the position of the test stimulus. In the dark-adapted preparation, we find no evidence of an inhibitory surround, so the responses measured are assumed to be central responses. Figure 3 also shows the ERF/A. There is a local dip in sensitivity of $1.1 \mathrm{log}$ units when the test is on top of adaptation. However, as the test spot moves away from the adaptation spot, the desensitizing effect decreases. At the position labelled $6^{\circ}$, for example, the effect is $0.6 \log$ units and at $3^{\circ}$, it is $0.2 \mathrm{log}$ units. Thus, the results shown in Figs. 2 and 3 indicate that the adaptation pool and the excitation pool determining the receptive field center are not coincident; adaptation spreads over a smaller arca than excitation. Figure 4 shows this result on another "off" unit. In this case, however, two ERF/A's were measured with the adapting spots at the two positions indicated on the graph. The adapting spot on the right produces a depression in sensitivity on the right side of the receptive field. Moving the adaptation spot $1.5^{\circ}$ to the left, keeping its intensity unchanged, produces a very different result; the dip in sensitivity occurs in the center of the receptive field. These findings are typical of the results found for rat retinal ganglion cells. Thirty-one out of 34 "off" units tested in this way showed a local dip in sensitivity at the position of the adapting spot.

These findings demonstrate that adaptation extends over a smaller area than excitation. Therefore, the site of adaptation cannot be at, or proximal to, the site of excitation summation. They do not, however, exclude the possibility of all adaptation taking place in the photoreceptors.

Ricco's law summation areas for excitation and adaptation

Here we report that, for the rat in contrast to the cat (Enroth-Cugell and Shapley, 1973), an adapting spot affects the receptive field locally. Can the apparent differences between the results from the cat and rat experiment be resolved? A partial answer may be 

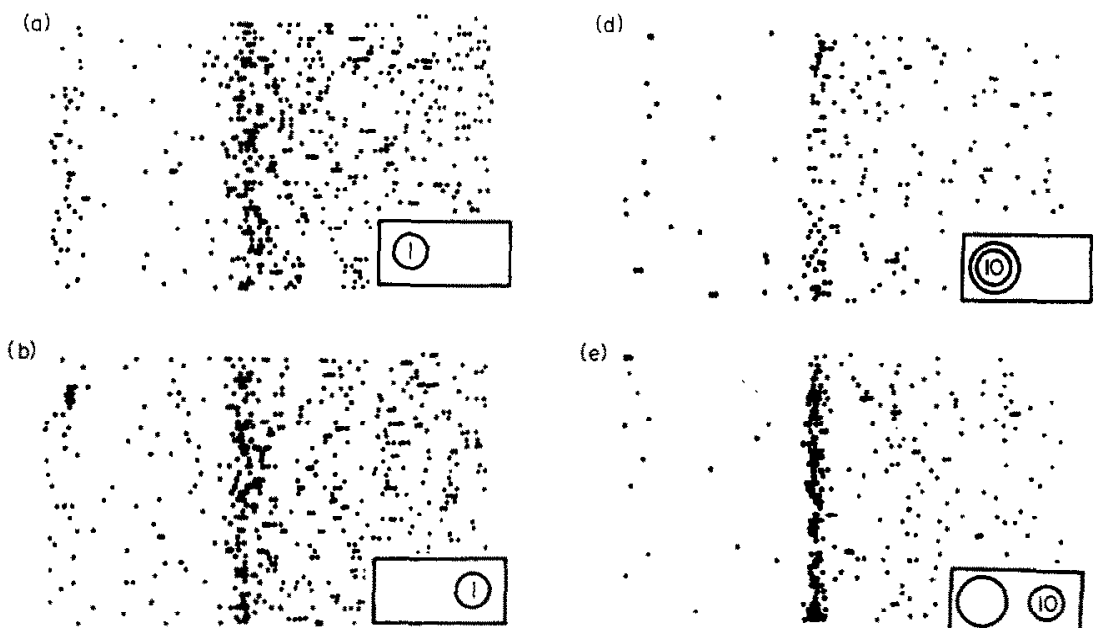

(e)

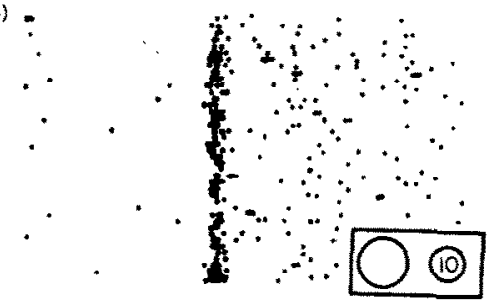

(c)

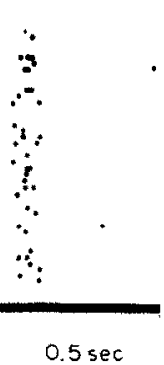

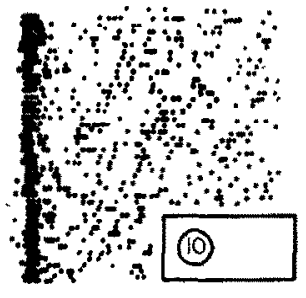

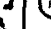

(f)

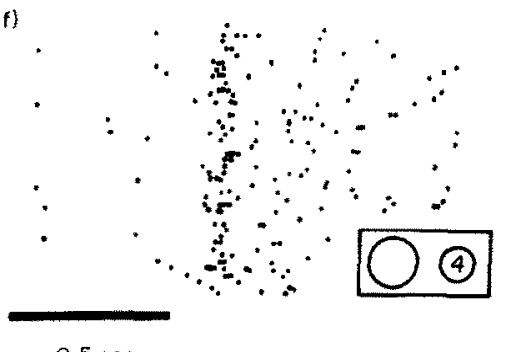

Fig. 2. A set of dot-displays obtained from two equally sensitive positions in the receptive field of an "off" unit. The insert in the lower right of each dot-display diagrammatically shows the stimulus configurations. The smaller circle represents the test spot. The number within it relates to its intensity relative to threshold. The larger circle represents the adapting spot. The intensity of the $\left(1^{\circ}\right)$ adapting spot does not change after it is initially adjusted. For example, the dot-display labelled "d" was obtained with a ten-times threshold $\left(1^{\circ}\right)$ test spot and a superimposed adapting spot, both on the left. When the test spot is moved to the right (e), the unit responds more vigorously than when it is superimposed (d).

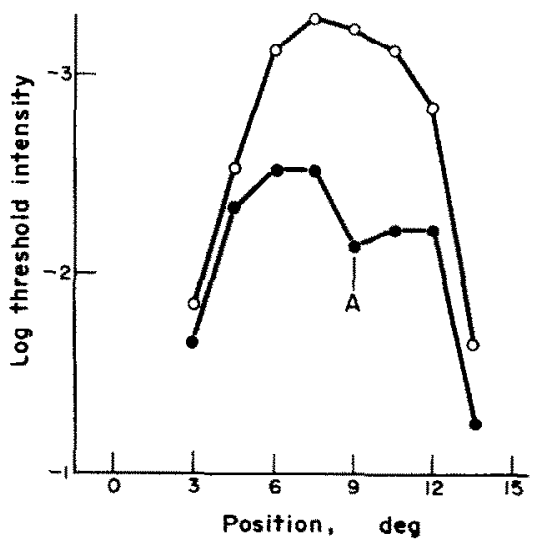

Fig. 3. Comparison between the open circles representing the excitatory receptive field sensitivity profile (ERF) of an "off" retinal ganglion cell and the filled circles representing its adapted sensitivity profile (ERF/A). The data points show how the relative intensity of a small test spot had to be varied as a function of position in the receptive field to produce a "threshold" response from the ganglion cell. The position marked $0^{\circ}$ is on the far right side of animal's receptive field. The position of the small $\left(1^{\circ}\right)$ adapting spot is indicated. Same unit as in Fig. 2.

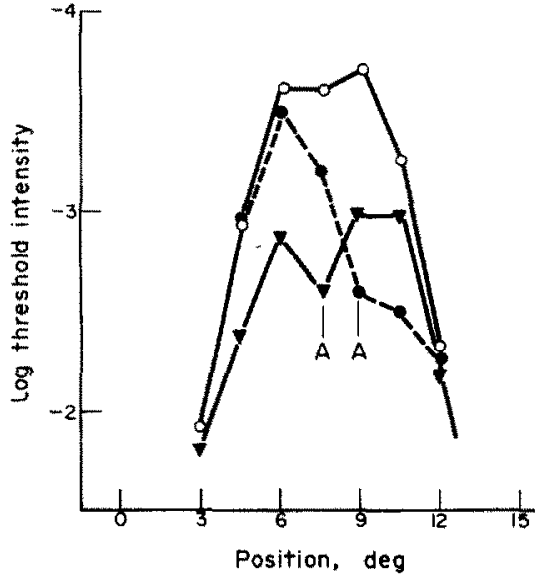

Fig. 4. Results similar to Fig. 3 from another "off" retinal ganglion cell. Receptive field sensitivity profiles with (closed symbols) and without (open circles) an adapting spot are shown. The closed triangles are for the adapting spot in the center of the receptive field and closed circles are for the adapting spot moved to the experimenter's right by $1.5^{\circ}$. 


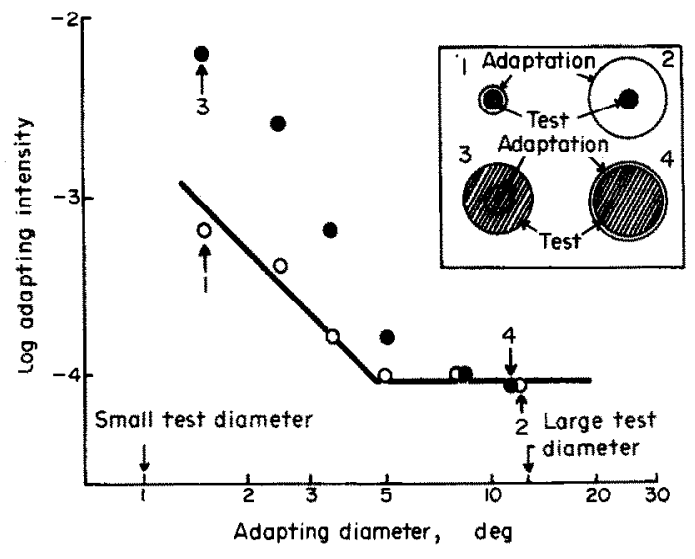

Fig. 5. Ricco's law summation areas for adaptation were measured by increasing the adapting spot size and using either a ten-times threshold small test (open circles) or a ten-times threshold large test (closed circles). The points give the adapting spot intensities which reduced the tentimes threshold tests to threshold. At each adapting diameter the measurements were obtained successively for the small and the large test spot. The small adapting spot is less effective when the test spot is large than when it is small. Adaptation is local.

found in Figs. 5 and 6 , of which the former shows a Ricco's law experiment measured with a small test spot and an adapting spot of various sizes. The open circles give the intensity, as determined by auditory thresholds, of the adaptation which reduced the $1^{\circ}$, ten-times threshold test spot to threshold. When measured in this way, the adaptive summation area is about $5^{\circ}$ dia which is quite a bit larger than would be predicted from the local dip produced in this same unit with a small adaptation spot (Fig. 6). In fact, $5^{\circ}$ is about the size of the Ricco's law summation area which would be expected if adaptation were summated according to the receptive field sensitivity profile, the result reported for the cat. Thus, there appears to be no conflict of data between our Ricco's

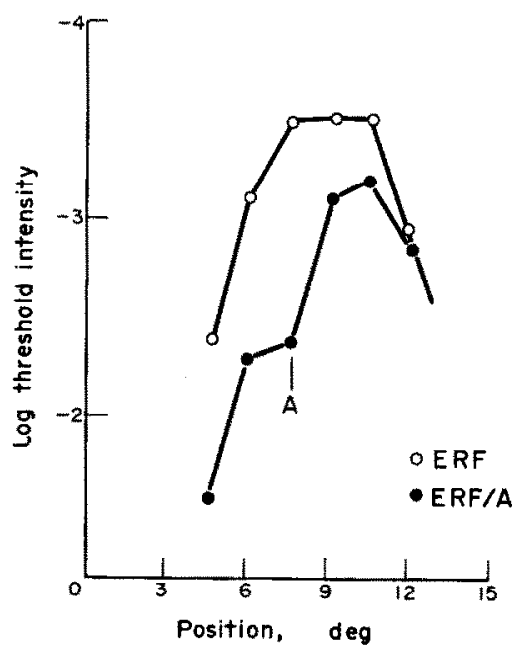

Fig. 6. Comparison between the excitatory receptive field sensitivity profile (open circles) of an "off" unit and its adapted sensitivity profile (closed circles) on the same ganglion cell of Fig. 5. The local dip in sensitivity at the location of the small $\left(1^{\circ}\right)$ adapting spot shows that adaptation spreads over a smaller area than does excitation. law experiments on the rat and the results of others on the cat.

Yet, as is clearly shown by the local effects of a small adapting spot (Fig. 6), the adaptive spread function cannot be identical to the sensitivity profile. To demonstrate that adaptation and excitation have different spread functions, it is only necessary to repeat the experiment using a large test spot rather than a small test spot. A small adapting spot will directly illuminate only a proportion of the receptors upon which the test flash falls. On the other hand, a large adapting spot will illuminate all of the receptors being tested. If adaptation is local, the small adapting spot should be relatively less effective than the large in desensitizing the large test spot. This result is shown in Fig. 5 by the filled circles which were determined using a large $12^{\circ}$, ten-times threshold test.

\section{Neural spread of adaptation}

Some of the strongest evidence for physiological spread of adaptation comes from experiments in which the minimum amount of light necessary to desensitize is determined. The idea behind these experiments is not new; it was used by Rushton (1965b) to argue, from psychophysical observations, for adaptation pools in man. So many of our ideas about sites of adaptation have changed in the last few years that it seemed important to use this simple and compelling argument on retinal ganglion cells of the rat. The objective of the experiment is to show an adaptive effect with a brief flash so weak that only a small percentage of the rods have caught a quantum of light. Even if the absorption of a single quantum of light by a rod totally desensitized it, at these extremely weak levels of illumination, this would cause only a small change in threshold. If, for example, one rod in ten absorbed a quantum, then the maximum effect which can be explained by receptor desensitization would be a $10 \%$ increase in threshold on the average. If one finds a larger effect than this, it indicates that the receptors which absorbed quanta must be influencing the thresholds of neighboring rods which have not. That is, adaptive signals must have spread laterally across the retina.

An optic tract unit was isolated and its receptive field center was located on a tangent screen. A test flash was centered in the receptive field. The intensity was then adjusted until the stimulus produced a small, but clearly detectable, change in spontaneous firing. A large, steady adapting stimulus, also centered in the receptive field, was, in turn, adjusted in intensity until it minimally adapted the test flash. Then, using these background and test stimuli, the changes in sensitivity following a sudden presentation of the background were measured. Since the response of the ganglion cell varies randomly from presentation to presentation, multiple presentations of the stimulus are required. In computing the number of rods which have absorbed quanta, to be conservative one must count all the quanta in the multiple presentations. First, the unadapted responses of the unit to 1015 presentations (one every $2 \mathrm{sec}$ ) of the test light without a background were displayed as dot patterns as in Fig. 1. Then, just before the start of the next test flash, the background was turned on and the impulse trains from 10 to 15 presentations of the test flash 
with a background were registered as a second dot display. We found in every case that the response was adapted. Moreover, as well as could be determined, the response to the first test Hash after the background onset was no stronger than the response to the tenth or to the fifteenth. There was never any trend with time toward progressively greater losses in sensitivity. Thus, the immediate effect of the background field was as strong as its more long-lasting components.

For the most adaptable units, the luminance of the adapting background was $1.6 \times 10^{-5} \mathrm{ft}-\mathrm{L}$ or $5.4 \times 10^{-5} \mathrm{~cd} / \mathrm{m}^{2}$. Taking into account the area of the pupil $\left(0.78 \mathrm{~mm}^{2}\right)$, the photopic to scotopic conversion for a $6000^{\circ} \mathrm{K}$ source $(2.4)$, the retinal illumination produced by the background was $1 \times 10^{-4}$ scotopic td. In man, 1 scotopic td of $500 \mathrm{~nm}$ produces a quantum irradiance on the retina of $5 \times 10^{6}$ quanta $/ \mathrm{mm}^{2}$ sec. The posterior nodal distance of the rat eye is $2.97 \mathrm{~mm}$ (Block, 1969) rather than $16.7 \mathrm{~mm}$ in man. Since retinal illuminance depends on the ratio of the squares of the posterior nodal distances (Le Grand, 1957 ), one arrives at 1 scotopic td $=1.6 \times 10^{8}$ quan$\mathrm{ta} / \mathrm{mm}^{2} / \mathrm{sec}$ (for rat). Assuming no losses in the ocular media, this adapting background therefore produces a shower of $1.6 \times 10^{4}$ quanta $/ \mathrm{mm}^{2} / \mathrm{sec}$ or 0.04 quanta $/ \mathrm{rod} / \mathrm{sec}$, using the figure of $4 \times 10^{5} \mathrm{rods} / \mathrm{mm}^{2}$ (Cone, 1963). It is likely that no more than $25 \%$ of this incident light is absorbed, so the background produced a flux of 0.01 quanta absorbed $/ \mathrm{rod} / \mathrm{sec}$, or less.

Thus, by the time the first flash was presented, about one rod in every 200 had absorbed a quantum of light and by the tenth, one out of every five had absorbed a quantum.

Figure 7 shows typical results from this type of experiment. Dot patterns from one of the most adaptable "off" units, out of the 15 studied, are displayed. The upper portion shows the unadapted response. The lower shows the responses to successive presentations of the test stimulus superimposed on the adapting stimulus. Even though this background produced a flux of only 0.01 quanta absorbed/rod/sec, it immediately desensitized the unit. The magnitude of the adaptive effect produced by the minimal background was determined by readjusting the stimulus intensity until the adapted response was identical with the unadapted, as determined by the dot-display. A five-fold increase $(0.8 \mathrm{log}$ units) in the test stimulus was required to restore the response to threshold. For the fifteen units tested, the minimal adaptive background produced, on the average, a flux of 1 quantum absorbed $/ 47 \mathrm{rods} / \mathrm{sec}$ and a change in threshold of $0.41 \log$ units (a factor of 2.6).

Figure 8 shows lateral spread of adaptation in still another way. A test spot was placed at the arrow to the right of the receptive field center. The test spot stayed fixed and a small $\left(1^{\circ}\right)$ adapting spot was moved across the receptive field. Our objective was to determine the adaptive sensitivity profile, the ARF. At each point, the intensity of the adaptation was adjusted to reduce a ten-times threshold test to threshold. The triangles show results of this experiment. As the adaptation was moved toward the receptive field center, its adapting effectivity increased. At the center of the field, where it is $3^{\circ}$ from the test, it was $0.4 \log$ units more effective than a superimposed adaptation.

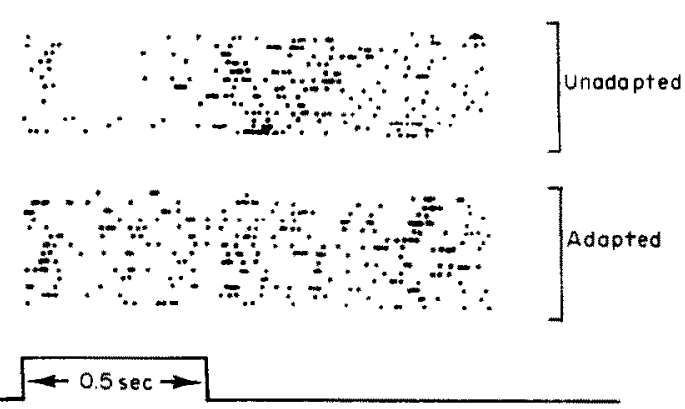

Fig. 7. The upper half shows response of an "off" unit to successive presentations of a slightly suprathreshold stimulus. The lower portions show the extent of desensitization in this unit when a weak adapting stimulus was presented. Although the adaptation produced a flux of only 0.01 quantum absorbed/rod/sec, a five-fold increase in the test stimulus was required to restore the response to threshold.

Scattered light from the adapting spot cannot explain the greater effect of the laterally-placed adapting spot, since the scatter intensity should be greater near the scatter source. However, the scattered light from the peripheral test spot also needs to be considered. If the scatter from the test onto the more sensitive center were exciting the ganglion cell centrally, then the greater effect of the centrally-placed adapting spot could be explained without invoking neural spread of adaptation. However, this cannot be the explanation. Our evidence on this point comes from the receptive field sensitivity profiles measured

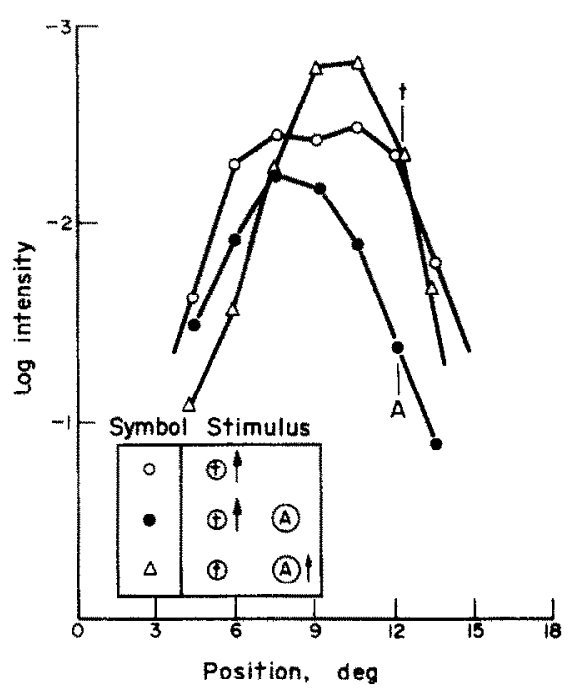

Fig. 8. The receptive field profile (ERF) as compared to the adaptive sensitivity profile (ARF) and the receptive field profile with a small adapting spot placed at the vertical line (ERF/A). The inserts explain the stimulus configurations. An arrow beside either test (t) or adaptation (A) indicates it varied in position and intensity. The receptive field profile was measured in the usual way with a $1^{\circ}$ roving test spot. The ARF was measured with a fixed test spot and a roving $1^{\circ}$ adapting light whose intensity was adjusted to reduce the ten-times threshold test to threshold. The ERF/A was measured as described before. Both the narrowness of the ARF profile and the local dip in the ERF/A indicate that adaptation spreads over a smaller area than does excitation. 
with and without an adapting spot at the test site. The closed circles (Fig. 8) show the receptive field sensitivity profile measured with a roving test and a fixed adapting spot in the same position occupied by the fixed test in the ARF measurements.

If light scatter from the test were exciting the center of the receptive field, then light from the adaptation should also scatter to the center where it would be expected locally to adapt the center, as in the previous experiments. This does not happen. The peripheral adapting spot to the right of center produces a local dip on the right side of the receptive field, with minimal change in the ERF centrally (closed circles, Fig. 8). Thus, while we find the adaptation affects the receptive field locally, the greater adapting influence of the laterally-placed adapting spot provides good evidence for neural spread of adaptive signals.

\section{DISCUSSION}

All our evidence (Green and Tong, 1974, 1975; and the present study) and that of others (Easter, 1968; Burkhardt and Berntson, 1972) indicales that adaptation spreads laterally with a sharper fall-off than the spread of excitation. Spread of light due to optical factors would be expected to cause some lateral spread of adaptation, and we set out to clarify the extent to which spread of light adaptation was physiological as opposed to optical in nature.

Lipetz (1961) projected a small conditioning spot onto the retina of the bullfog for several min. He then measured ganglion cell thresholds for a test stimulus at the previously illuminated area and at an unilluminated area. The unilluminated area showed an increase in threshold which he argued was greater than could be explained by scattered light. He concluded that adapting a subset of the receptors in a ganglion cell's receptive field reduced the efficiency of excitation transmission from receptors anywhere in the receptive field. The magnitude of the threshold change was found to be directly proportional to the sensitivity of the region of the receptive field illuminated by the conditioning exposure. $A$ larger adapting effect with the test and adaptation separated, rather than coincident, seemed to demonstrate spread in a way which could not be due to scattered light.

Easter (1968), however, has offered an alternative explanation for this result. When the test stimulus is not on the plateau of the receptive field sensitivity profile, it might be outside the boundaries of the ganglion cell receptive field and might be affecting the receptive field through scattered light. Even if the light exclusively affected the sensitivity of photoreceptors, the displaced adapting spot at the actual site of excitation would be more effective in desensitizing the ganglion cell than the adapting spot coincident with such a test spot. Thus, the Lipetz result in itself does not prove there is neural spread of adaptation.

To circumvent these difficulties, Easter tested equally sensitive areas in the receptive field of goldfish retinal ganglion cells. Under these conditions, he found that an adapting spot desensitized most when it was coincident with the test spot, a result which by itself is consistent with the hypothesis that scattered light totally mediates lateral spread of adaptation.
With rat retinal ganglion cells, we find that an adapting spot in the center of the receptive field can be more effective than one placed on top of a peripheral test spot, a result similar to Lipetz's (1961). The possibility that scattered light caused the effect was eliminated by demonstrating that the peripheral test spot was stimulating the periphery of the ganglion cells receptive field center. This was done by showing that when the flashing test spot was replaced with a steady adapting spot, the adapting spot maximally desensitized the field directly under its geometric image.

Working on the cat retina, Enroth-Cugell and coworkers find that the Ricco's law summation areas for excitation and adaptation are the same. They use this to conclude that the state of adaptation of ganglion cells is determined by the spatial distribution of light flux weighted by the receptive field sensitivity, independent of how the flux is distributed over the retina. Burkhardt and Berntson (1972), using small adapting spots, found evidence for spread of adaptive effects which were narrower than frog retinal ganglion cell's excitatory receptive field. Rather paradoxically, measurements of the adapting and excitatory effects of concentric spots of variable radius, a Ricco's experiment, yielded similar radius-sensitivity curves. We have found a similar result for the rat (see Fig. 5). However, in another kind of Ricco's law experiment, a small adapting spot less effectively desensitizes the larger of two tests. There may be confounding factors contributing to this kind of experiment since the small and large test spots may stimulate the center and surround mechanisms to different extents. We have not tested for these kinds of effects. Nonetheless, the important point is that a Ricco's law experiment using a small test spot and adapting spots of varying size may not effectively reveal differences in the pooling of adaptive and excitatory signals. Clearly, field adaptation must be quite complex.

Given the discrepancy between our conclusions about adaptation in the rat and those previously mentioned for the cat, it is worth pointing out that our results are consistent with other research that indicates that the excitation and adaptation pools are different (Easter, 1968; Burkhardt and Berntson, 1972). We know that the b-wave shows all aspects of adaptation. The increment threshold curves for b-wave threshold are very much like their psychophysical counterparts; they follow the same laws over a broad range (Dowling, 1967). Therefore, the site of visual adaptation must be distal to the generation of the b-wave. This means adaptation occurs before signals converge onto the ganglion cells. If any additional convergence occurs at ganglion cells, then it is not possible for the ganglion cell's ordinary excitatory receptive field to be the same as the adaptation pool; one expects spread of adaptation to be local. However, the important point is not the differences we find between cat and rat retinal ganglion cells, but that both sets of experiments provide good reason to believe that spread of adaptation is at least in part neural.

The most direct evidence for physiological spread of adaptation comes from the experiments on the minimum amount of light necessary to desensitize the ganglion cells. Only a small fraction of the rods need 
absorb a quantum of light to cause a severd-fold change in sensitivity. The only way this can occur is through neural spread of adaptive signals across the retina.

What our results show is that in the rat, adaptation spreads at a site which is distal to the site of excitation summation. The question that remains unanswered is: how far distal? In particular, are signals spreading from one photoreceptor to another? While there is nothing in our experiments which logically excludes this possibility, the avalable evidence suggests that this is not the case. Interreceptor contacts have not been seen between rat rods. Moreover, out finding that an adapting spot over $100 \mu \mathrm{m}$ away from a test spot can be more effective than one superimposed is relevant, for it implies that a quantum falling to the side of a photoreceptor can have a greater adapting effect than a quantum falling directly on it. This is a very unlikely effect to be mediated by interreceptor interactions. On these grounds, we suggest that the site of low level adaptation is proximal to the receptors, but distal to the ganglion cells.

The findings reported here help to put the previous experimental studies into proper perspective by showing rats, goldfish, and frogs have similar adaptive properties. While some recent electrophysiological studies have emphasized the importance of the photom receptors in the process of visual adaptation, even in the skate retina where most of the adaptation occurs in the photoreceptors (Dowling and Ripps, 1971, 1972), both receptor and network mechanisms are important in visual adaptation (Green, Dowling. Siegel and Ripps, 1975). Moreover, all of the available evidence to date seems to suggest that changes in sensitivity at the lowest levels of adaptation are controlled exclusively by changes in the more proximal retina.

Acknowledgements-We would like to thank Dr. Edward Pugh for his helpful comments and assistance in designing the minimal adaptation experiments. This research was supported by USPHS grant EY 00379. Dr. Cicerone was supported by NEI posidoctoral fellowship EY 02161.

\section{REFERENCES}

Barlow H. B. and Andrews D. P. (1967) Sensitivity of receptors and receptor pools. $J$. opt. Soc. Am. 57, $837-838$.

Barlow H. B. and Andrews D. P. (1973) The site at which rhodopsin bleaching raises the scotopic threshold. Wision Res. 13, 903-908.

Baylor D. A. and Hodgkin A. L. (1974) Changes in time scale and sensitivity in turtle photoreceptors. J. Physiol. 242, $729-758$.

Block M. T. (1969) A note on the refraction and image formation of the rat's eye. Vision Res. 7, 705-71\%.

Bonds A. B. Erroth-Cugell C, and Pinto L. H. (1972) Image quality of the cat eye measured during retinal gang lion cell experiments. J. Physiol., Lond 220, 383-401.

Boynton R. M. and Whitten D. N. (1970) Visual adaptation in monkey cones: recordings of late receptor potentials. Science 170,1423 . 1425 .

Burkhardt D. A and Berntson G. G. (1972) Light adaptation and excitation: lateral spread of signals within the frog's retina. Vision Res. 12, 1095-1111.
Cleland B. G. and Enroh-Cugell C. (1968) Quantitatiwe aspects of sensitivity and summation in the cat retina. J. Phwiol. Lond. 198.17-38.

Cone R. A. (1963) Quantum relations of the rat clectroretinogram. J. gen. Physiol. 46, $1267-1286$.

Dowling I. E. (1963) Neural and photochemical mechanisms of visual adaptation in the rat. $J$ gen. Phy siol. 46, 1287-1301.

Dowling I. E (1967) The site of visual adaptation. Sciente $155,273-279$.

Dowling J. E. and Ripps H. (1971) S-potentials in the skate retina: intracellular recordings during light and dark adaptation. $J$. gen. Physiol. 58, 163 189 .

Dowling J. E. and Ripps H. (1972) Adaptation in skate photoreceptors. I. gen. physiol. 60, 698-719.

Easter $S . S$ (1968) Adaptation in the goldfish retina. $J$. Phystol, Lond. 195, 273-281.

Enroth-Cugell C and Shapley R. M. (1973) Flux, not retinal illumination, is what cat retinal ganglion cells really care about f. Physiol., Lond. 233. $311-326$

Glickstein M. and Millodot M. (1970) Retinoscopy and eye size. Stince 168, 605-606.

Grabowski S. R.. Pinto L. H. and Pak W. L. (1972) Adaptation in retinal rods of axolot: intracellular recordings. Stiknce 176, 1240-1243

Green D. G. (1973) Scotopic and photopic components of the rat electroretinogram. J. Physiol. Lond. 228, $781-\ldots 797$.

Green D. G. and Tong L. (1974) Lateral spread of light adaptation in the rat retina. Soc. Netrosci. Abstr. 4, 235 .

Green D. G. and Tong L. (1975) Spread of light adaptation in the rat retina. Anntal Meeting of Association for Research in Vision and Ophthalmology, p. 87.

Green D. G. Dowling J. E., Siegel I. M. and Ripps $H$. (1975) Retinal mechanisms of visual adaptation in the skate J. Gen. Phystol. 65, 483-502.

Hecht $S .(1937)$ Rods, cones and the chemical basis of cone vision. Physiol. Rev. 17, 239-290.

Hood D. C. and Hock P. A. (1973) Recovery of cone receptor activity in the frog's isolated retina. Vision Res. 13, $1943 \cdots 1951$

Hoyd D. C. Hock P. A. and Grover B. G. (1973) Dark adaptation of the frog's rods. Vision Res. 13, 1953-1963.

Kleinschmidt J. (1973) Adaptation properties of intracellularly recorded gecko photoreceptor potentials. In Biochemistry and Physiology of Vistal Pigments (Edited by Sanger H., Springer, Berlin.

Kleinschmidt J. and Dowing J. E. (1975) Intracellular recording from gecko photoreceptors during light and dark adaptation. I. gen. Physiol. 66, 617-648.

Le Grand Y. (1957) Light. Colour and Vision. Chapman \& Hall, London.

Levick W. R. (1972) Another tungsten microelectrode. Med. Biol. Engng 10, 510515 .

Lipetz. L. E. (1961) A mechanism of light adaptation. Science 133, 639-640.

Rushton W. A. H. (1961) Rhodopsin measurement and dark-adaptation on a subject deficient in cone vision. J. Physiol. Lond. 156, 193-205.

Rushton W. A. H. (1965a) Vision adaptation (Fernier Lecture). Proc. R. Soc. B, 162, 20-46.

Rushton W. A. H. (1965b) Sexsitivity of rods ander illumination. J. Physiol. Lond. 178, 141-160.

Rushton W. A. H. and Westheimer G. (1962) The effect on rod threshold of bleaching neighboring rods. J. Phy sianl. Lond. 164. 318-329.

Wiesenfeld $Z$ and Branchek T. (1976) Refractive state and visual acuity in the hooded rat, Vision Res. 16, 823-827,

Witkovsky P.. Nelson J. and Ripps H. (1973) Spectral properties of the isolated receptor potential of the carp retina. J. gon. Fhysiol. 61. 401-423. 